\title{
Epigenetics in Clinical Practice: Characterizing Patient and Provider Experiences with MTHFR Polymorphisms and Methylfolate
}

Erica Oberg ${ }^{1,2 *}$, Chris Givant ${ }^{3}$, Briana Fisk ${ }^{4}$, Carina Parikh ${ }^{2}$ and Ryan Bradley ${ }^{1,2,5}$

${ }^{1}$ Pacific Health in Practice, LLC. La Jolla, CA, USA

${ }^{2}$ National College of Natural Medicine, School of Research and Graduate Studies, Portland, USA

${ }^{3}$ La Vita Compounding Pharmacy, San Diego, CA, USA

${ }^{4}$ George Washington University, Milken Institute School of Public Health, Washington D.C, USA

${ }^{5}$ National College of Natural Medicine, Helfgott Research Institute, Portland, USA

\section{Abstract}

Background: Observational research associating methylenetetrahydrofolate reductase (MTHFR) polymorphisms with risk of autism, depression, cancer and cardiovascular disease has led to increased diagnoses of MTHFR, however, doctors lack knowledge about safety, effectiveness, and clinical implications of MTHFR treatment. Treatment strategies are hypothetical and mechanistically-based, including methylfolate with or without other B vitamins.

Aims: This study was designed to formally describe patient and healthcare provider experiences with the diagnosis and clinical management of MTHFR.

Methods: Guided by a structured interview guide, a qualitative study queried patients' and providers' observations regarding: testing indications, reaction to results, treatment protocols, and clinical response including adverse effects.

Results: Thirty patients and eight doctors participated. Patient themes included emotionality associated with diagnosis, classification of signs and symptoms, and challenges with treatment. They expressed confusion over their diagnosis, and frustration with the state of knowledge their providers had regarding MTHFR. Testing indications included: fatigue $(21 \%)$, hormone imbalances $(13 \%)$, and neurological symptoms $(13 \%)$ including brain fog $(8 \%)$. Patients reported improvements in physical $(60 \%)$ and mental/behavioral symptoms $(36 \%)$ following treatment. A minority of participants reported side effects but they occurred in almost every body system and ranged in severity. Doctors relied on trial and error to determine treatment doses, frequency and components.

Conclusions: MTHFR testing results in variable clinical processes in domains related to delivery of diagnosis and prognosis, and therapeutic options. However, patients report largely positive experiences. Clinicians and patients would benefit from therapeutic algorithms based on rigorous research.

Keywords: Methylenetetrahydrofolate Reductase; MTHFR; Methylfolate; B Vitamins; Nutrigenomics; Qualitative Research

\section{Introduction}

The 5,10-methylenetetrahydrofolate reductase (MTHFR) gene codes for the MTHFR enzyme, which catalyzes the conversion of 5,10-methylenetetrahydrofolate to 5-methylenetetrahydrofolate, i.e., it "reduces" folate. 5-methyltetrahydrofolate is the substrate for the remethylation of homocysteine to methionine, thus assisting in the metabolism of homocysteine and aiding in the recycling of methionine. Genetic polymorphisms in the MTFHR gene lead to reductions in the efficiency of methylation reactions, resulting in the accumulation of metabolic intermediates. Methylation defects, like those caused by mutations in MTHFR, hinder optimal function of essential metabolic pathways, including DNA synthesis and repair, neurotransmitter synthesis, gene regulation, protein function, antioxidant synthesis, heavy metal detoxification, immune activity, homocysteine regulation, and hormone activity [1]. Thus, the MTHFR mutation can lead to a wide range of metabolic dysregulation, for example, the accumulation of homocysteine, reduced methionine, and dysregulation of other pathways requiring methylation intermediates [2]. Because methylation reactions are ubiquitous and function directly in post-transcriptional epigenetic regulation and as an intermediate in numerous metabolic reactions, it is no surprise that MTHFR polymorphisms have been associated with a wide variety of symptoms and associated diseases, including depression, hypertension and occlusive vascular disease, dementia and Alzheimer's disease [3].

The two most common MTHFR gene mutations occur on alleles C677T and A1298C. For C677T, a thymine may replace cytosine at position 677 on one or both gene copies, leading to an alanine to valine substitution. For A1298C, adenine may be replaced by cytosine on one or both gene copies leading to glutamate substitution for alanine. A recent meta-analysis suggest that frequency of homozygous C677T (TT) allele presence is $8-18 \%$ in people of European ancestry, $21-25 \%$ in Hispanics, $7 \%$ in Black populations, and $11 \%$ in Asians [4]. Heterozygosity (CT) is much more common; $50 \%$ of Hispanics, $43 \%$ non-Hispanic whites, and $20 \%$ of non-Hispanic blacks have one mutation. Research suggests no significant difference in frequency between males and females, but that the mutation is more common in younger populations than the elderly.

*Corresponding author: Erica Oberg, ND, MPH, Pacific Health in Practice, La Jolla CA, USA, Tel: 858459 6919; 858459 6933; E-mail: erica.oberg@icloud.com

Received July 13, 2015; Accepted August 26, 2015; Published September 04, 2015

Citation: Oberg E, Givant C, Fisk B, Parikh C, Bradley R (2015) Epigenetics in Clinical Practice: Characterizing Patient and Provider Experiences with MTHFR Polymorphisms and Methylfolate. J Clin Med Genom 3: 124. doi: 10.4172/2472128X.1000124

Copyright: (C) 2015 Oberg E, et al. This is an open-access article distributed under the terms of the Creative Commons Attribution License, which permits unrestricted use, distribution, and reproduction in any medium, provided the original author and source are credited. 


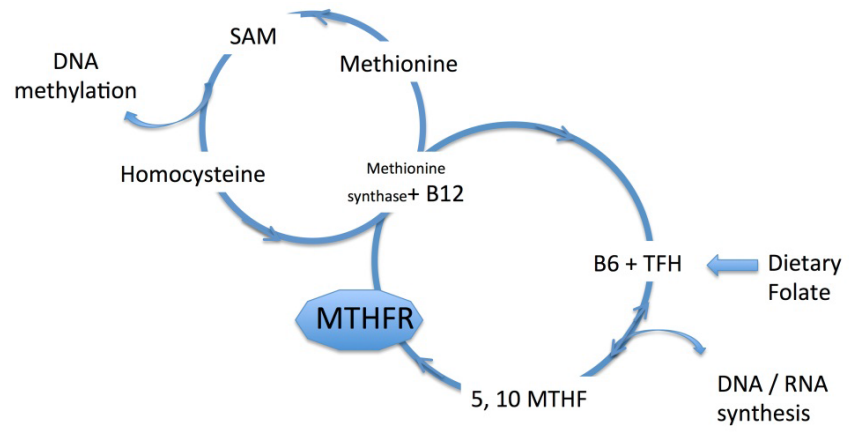

Figure 1: Biochemicalmethyl pathwayinvolving 5,10-methylenetetrahydrofolate reductase (MTHFR)

For the A1298C allele mutations, frequencies are estimated to be quite low. Only 3-10\% of the US population is homozygous for A1298C (CC) with $29-42 \%$ heterozygous depending on ethnicity. Additionally, there is a $15-20 \%$ frequency of C677T/A1298C compound heterozygosity. Functionally, C677T heterozygotes have about $65 \%$ of the MTHFR enzyme activity while TT homozygotes have about $30 \%$ compared to homozygous CC individuals [5]. Figure 1 illustrates the biochemical methyl pathways in which MTHFR enzyme activity is a rate-limiting step.

Known associations between MTHFR mutations and physical, mental, emotional symptoms

Several epidemiologic case-control studies demonstrate significant associations with MTHFR homo- and heterozygous genotypes, mental and physical symptoms, as well as increased risk of diseases ranging from heart disease to schizophrenia to cancer [6,7]. However, not all studies have found associations. It appears that this may because the MTHFR genotype-disease risk is confounded by serum folate status. A number of studies suggest that the increased odds ratios of disease are only observed in populations with insufficient serum folate levels $[6,8]$.

Mental: Regarding mental health, C677T polymorphisms have been associated with higher stress reactivity, increased severity of depression, and decreased general mental health status $[9,10]$. Levels of homocysteine and rates of schizophrenia and anxiety have been found to be significantly higher in those with $\mathrm{C} 677 \mathrm{C} / \mathrm{T}$ heterozygosity $(\mathrm{r}=-0.58 ; \mathrm{P}<0.01)$. [7] The C677TT compared to CC genotypes have been associated with increased odds of unipolar depression (OR: 1.36, 95\% CI 1.11-1.67), schizophrenia (OR: 1.44, 95\% CI 1.21-1.70), and bipolar disorder (OR: 1.82, 95\% CI: 1.22-2.70) [11] ; as well as autistic spectrum disorders $(\mathrm{OR}=2.26,95 \% \mathrm{CI} 1.30-3.91)$ [12].

Physical: In the domain of physical health, perhaps the most significant and widely known effect of MTHFR genotype polymorphisms is the well-observed 1.8 to 2 -fold increased risk of neural tube defects with fetal or maternal 677TT genotype that interacts with levels of erythrocyte folic acid [4]. Along with this, a 3-fold increased risk of pre-eclampsia is seen in North American populations with 677TT. Since folic acid fortification of food has become widespread around the world, there has been an estimated $46 \%$ reduction in the prevalence of neural tube defects since 2008[6]. It has been suggested that methylfolate treatment would be a superior preventive strategy given that it is the predominant natural form, readily available for transport and metabolism and bypasses the enzyme defect present in people with the MTHFR genotypes of note. However, this form of the vitamin has not been tested in clinical trials [13].
The association between C677T genotype and elevated homocysteine is a health effect of the MTHFR gene generally appreciated by cardiologists. However, data from the National Health and Nutrition Examination Survey (NHANES) cohort suggest the MTHFR 677TT genotype was associated with a lower rate of CVD mortality following routine dietary fortification of grains with folic acid. Further, supplementation with B vitamins, while effective in lowering homocysteine, does not appear to reduce cardiovascular mortality except in the case of a small benefit in stroke [14]. Homocysteine does not appear to increase in A1298CT/CC unless it is compounded with a C677T mutation [15].

The MTHFR A1298C genetic polymorphism is associated with increased risk of ischemic stroke, compared to 1298AA homozygosity [16]. The 677TT genotype was associated with an increased risk of stroke only in geographic regions of low folate. In populations of optimal folate status and in regions of mandatory folic acid fortification, the effect of the TT homozygosity on stroke risk was not present [14], despite a slightly increased rate of hypertension even in heterozygous populations [17]. These associations reinforce the importance of not only the genetic status of an individual but also the nutritional epigenetic environment that influences gene expression.

Associations between increased cancer risk and MTHFR polymorphisms (both C677T and A1298C) have been observed in a number of cancer types in geographically and ethnically diverse populations. Underlying this observation is evidence of functional hypomethylation. Global DNA hypomethylation is implicated in the early pathogenesis of cancer and these studies directly measured methylation in subjects with cancer as compared to matched controls. Based on this, a threshold of hypomethylation was determined, which correlated with genotype $[1,18]$. Odds of cancer are reported to increase up to 7 fold in homozygous C677TT subjects with insufficient folate intake [19].

\section{Lack of experimental evidence on the need for treating MTHFR mutations}

Despite the abundance of epidemiologic studies, clinical trials are limited. In the early 2000 s, a series of trials examined the effect of folateenhancing dietary interventions on plasma folate and plasma total homocysteine with respect to MTHFR 677T genotypes and found that subjects with homozygous mutations required higher doses to achieve equivalent homocysteine reductions $[19,20]$. This finding has been replicated by others as well [21]. Of course, a cardiovascular benefit of homocysteine lowering with folic acid, even in the presence of C677T mutations has not been found, with the exception of stroke [14].

A 2011 trial of folic acid supplementation of women undergoing in-vitro fertilization found an increased rate of twins that correlated with serum folate status irrespective of genotype. Women with 1298CC mutations were less likely to conceive or produce a live birth [22]. In the domain of mental health, a trial of folinic acid in SSRI-refractory depression found modest benefit [23].

Despite the lack of experimental evidence, the available observational data combined with the availability of direct to consumer laboratory testing for MTHFR mutations, has led to the generation of a significant "crowd sourced" library of un-validated information on the internet [24]. Although some of this information comes from self-proclaimed physician experts, much of it is sourced directly from consumers describing their experiences during personal experimentation with treatments. Therefore, given the rising popularity 
of MTHFR testing and potential for misinformation on the significance of MTHFR polymorphisms and effective "treatment" strategies, there is an emerging need for structured data collection of all types in order to inform clinicians and the public about the various implications of MTHFR-related genetic findings.

We conducted a formal qualitative study in order to understand patient and health care provider experiences with MTHFR mutations and various treatment strategies, and to present their observations in a more structured manner than any currently available information.

\section{Methods}

\section{Selection of participants and recruitment}

Participants were doctors with clinical experience using methylfolate, and patients with personal experience using methylfolate, as a clinical intervention for MTHFR polymorphisms. Following IRB approval, doctors were recruited from a convenience sample selected from the professional contacts of the study team, as well as, from prescriber records from a compounding pharmacy that prepares an injectable methylfolate. Doctors included medical (MD), osteopathic (DO) and naturopathic (ND) doctors.

Patients who had been prescribed injectable methylfolate were then identified from pharmacy records. Candidate participants were sent letters of invitation, to which they responded at their own discretion. Upon response, personal experience with methylfolate and medical diagnosis of MTHFR polymorphism was confirmed over the telephone, at which time the candidate was scheduled for one of four focus groups or a telephone interview if they were unable to attend a group. A small stipend was provided for the time and travel required of participants.

\section{Focus groups and interviews}

Two structured interview guides, including scripts, were prepared to include open-ended questions regarding the relevant domains of interest for the doctors and patients. We drew upon the Health Belief logic model to ensure relevant topics were covered in the interviews [25]. The Health Belief Model is a well-established model developed to explain and predict health-related behaviors, particularly in regard to the uptake of new health practices. For the patients, the interview guide included queries in the following areas: data source for their MTHFR status and how it was communicated to them; their current treatment protocol; experience with injectable methylfolate compared to other treatments; and changes in their symptoms during treatment. For the doctors, the interview guide included queries in the following areas: how do they identify genetic polymorphisms including MTHFR; their education background in the area of nutrigenomics; basic and detailed elements of their treatment protocols; and their observations regarding changes in symptoms.

All interviewers were trained on the use of the interview guides and the interview script prior to the focus groups. Three qualitative researchers conducted the interviews and focus groups. Focus groups were approximately 90 minutes and interviews ranged from 25 to 60 minutes. Ultimately, thirty patients and eight doctors participated in the focus groups. Focus groups and interviews were audio-recorded and transcribed by a professional transcriptionist. Transcripts were imported into DeDoose ${ }^{\infty}$ (Los Angeles CA), qualitative software that assists with coding and data management.

\section{Analysis}

We used an inductive/deductive content analysis approach [26].
We first abstracted information pertaining to the key elements in the interview guide and developed an a priori code list based on the topics of inquiry. A second researcher reviewed the code list and a final code list was determined by consensus, yielding 130 unique codes. All 3 researchers then coded transcripts separately. DeDoose mapping and co-occurrence features were used to aggregate codes into themes, eliminating responses that were off-topic or were isolated opinions not expressed by at least 3 participants. Redundant responses and shared opinions were aggregated into super-codes and sorted by code density as well as by participant ID (to account for repetitive statements by individual participants). The analysis team used an iterative process to discuss the themes, clarify and expand upon interpretations of findings, and contextualize the coded responses back into the transcripts of the full conversation. Concepts and themes were also organized by participant ID to quantify the relative frequency of descriptors of the participants' experience regarding a priori specified domains of interest. Pertinent, illustrative quotes were selected to convey authentic experiences.

\section{Results}

\section{Overview}

The major themes included 1) characteristics of clinical treatment 2) reactions to diagnosis, 3) reports of physical and mental symptom changes, and 4) side effects and adverse events. Characteristics of treatment protocols were aggregated and found to be highly heterogenous; no dominant or consistent strategy was identifiable. When patients reported the emotional experience of receiving their diagnosis and beginning treatment, a majority of patients described it as life-changing, validating, and positive. Patients demonstrated significant confusion over their actual diagnosis. The most common presenting symptoms, in descending order, were: fatigue $(21 \%)$, hormone imbalances (13\%), neurological symptoms (13\%), digestive disturbances (10\%), and brain fog (8\%). Many positive improvements in symptoms were in domains of mental-emotional health. Side effects occurred in a minority of participants but ranged in severity and occurred in almost every body system.

\section{Patient experiences - A life-changing event and sense of validation}

Patients consistently reported that the diagnosis and treatment of MTHFR polymorphisms was a life-transforming event, however a few patients had neutral opinions regarding the impact of their MTHFRrelated diagnosis. The relative frequency of reactions to diagnosis were: relief $(35 \%)$, a sense of hopefulness $(31 \%)$, reflection in the impact on family members (26\%), a sense of empowerment (19\%), frustration (17\%), confusion/feeling overwhelmed (15\%), excitement (9\%) and indifference (4\%). Many patients reported their experiences with lifelong symptoms, working with countless doctors, and years of frustration at the lack of explanation or definitive diagnosis. Table 1 captures a representative selection of patient quotes regarding the impact MTHFR diagnosis and treatment had on their lives (Table 1).

About a third of the code density clustered in the domain of 'patient's reactions to diagnosis' also reported the impact their diagnosis on family members. "I was a little freaked out, and of course, I had to spread it to my whole family, and you know, not knowing what it was, I think that I caused a little bit of hysteria amongst my family members as well for the time being." Patients reported a wide range of reactions from their family members regarding their interest in testing- some refused, while others were highly motivated. 
"I feel like I get a chance to be on par with everybody else. I feel like I missed out on a lot of activities because of the fatigue" "It's like hallelujah. I'm a believer. That's all I have to say."

"I freaked out [upon diagnosis]. I felt: oh no, there's something terribly wrong with me so I looked it up online and read everything I could about it, and you know, I just had never... I was reading about naturopathic doctors who are doing genetic testing, and so I was interested in it, but I had never heard of this particular one, and so then I felt good. Now I know where we're going with this, and so, it isn't as bad as I thought it was. I mean the thing of it is that I just cannot believe that methylfolate and methylcobalamin took away all the symptoms that I had for 6 years... I could not believe it".

"[I've had] fibromyalgia for 30 years, and [my doctor had] decided to try and tackle that. He's seen several different problems rolled into one so he tested me for the MTHFR gene and I am homozygous for the C677T mutation, and he gave me the combination of the methylfolate and methyl B12. I didn't really feel that much difference the first time, but we tried it again, and then all of a sudden, I realized I had a lot more energy, and so l've been giving myself injections twice a week for the last, almost 2 years: a year and 9 months. It's giving me my life back, miracle"

"I have noticed no side effects, but I would probably put up with a lot of symptoms to have this much energy again."

"[lt has been] maybe since I was 15 or so is when I can recall having more troubles concentrating in school ... when I was 30 , I started having real problems of depression, but now I think -- I have a whole realization that, like wow, life's not something I have to just get through."

"I was excited that there was an answer. I felt so relieved that there was going to be a new change for my life."

"[The diagnosis] really wasn't all that surprising to me... I had been feeling kind of rundown...and I got tired a lot... I was almost glad to find out what it was and that I could do something about it... Going gluten free made me feel better, but it hadn't made my energy come back entirely... I didn't even know what it was like to feel good for the longest time"

"It answered so many questions from my entire life. The symptoms that I had from the time I was a child, so I already knew, but it was a relief. It was like I finally have an answer and I have a hope for a lot better life"

Table 1: Patients' quotes about the life-changing nature of diagnosis and treatment

\begin{tabular}{|c|c|}
\hline Theme & Patient Quotes \\
\hline \multirow[t]{3}{*}{$\begin{array}{l}\text { Validation after "regret" } \\
\text { (frustration/anger/feeling } \\
\text { of being cheated prior to } \\
\text { diagnosis) }\end{array}$} & $\begin{array}{l}\text { "....people were telling me it's all in your mind, you know. It's all in your mind. You just need to meditate and you'll be fine. You should see } \\
\text { the counselor... I'm a really happy person... my life was going well. I have this awesome relationship. My job was great... What can I be } \\
\text { upset about? But that I found out...this [MTHFR status] really had a lot to do with it, so I thought: yay okay, now we have a direction to go" }\end{array}$ \\
\hline & $\begin{array}{l}\text { "I have three different doctors, and my God, I tell them the diagnosis; I found an answer! And they basically were like well, that's great } \\
\text { for you; keep doing what you're doing, but that isn't anything that we treat or know anything about. You know, [it felt like] a brush off, like } \\
\text { you're on your own" }\end{array}$ \\
\hline & "I feel cheated that I couldn't have had a more comprehensive exam much earlier, that no one really took me seriously" \\
\hline \multirow[t]{4}{*}{$\begin{array}{l}\text { Validation following years } \\
\text { without diagnosis }\end{array}$} & $\begin{array}{l}\text { It wasn't in my head and I wasn't depressed. I didn't need an anti-depressant or whatever. That's what everybody kept trying to give me. } \\
\text { I knew there was something wrong. I wanted the answer, but it wasn't [until this diagnosis] was there the answer." }\end{array}$ \\
\hline & $\begin{array}{l}\text { "I had a stroke. Prior to the stroke I was a certified personal trainer. I was the healthiest person in my family. I took care of myself, and I } \\
\text { was the only one in my family that got a stroke, and I always wondered why me?." }\end{array}$ \\
\hline & $\begin{array}{l}\text { "I felt a little less crazy because I've always been a little different and there's a lot of other symptoms. When I looked up what the } \\
\text { homozygous mutation meant and some of the symptoms that come along with it, it all made sense, so it was sort of a relief for me and I } \\
\text { was happy that okay, there's something that we can actually address and deal with." }\end{array}$ \\
\hline & $\begin{array}{l}\text { "I actually found it comforting and a relief. I have had anxiety and panic attacks, you know, for years, and when I looked up to what the } \\
\text { symptoms were, you know, I thought oh, okay, this explains a lot as well as having, you know, IBS and GERD, and you know, all those } \\
\text { other fun things, so to me, it was really a relief." }\end{array}$ \\
\hline \multirow[t]{3}{*}{ Empowerment } & $\begin{array}{l}\text { "I wasn't shocked or upset by it all. I just felt like okay, that's another thing... I felt awful every day, so I think... maybe here's something } \\
\text { that we can do that will actually make a difference" }\end{array}$ \\
\hline & $\begin{array}{l}\text { "I think just having a diagnosis and knowing there's a cause and there's a treatment, that's huge. It took the mental burden of what the } \\
\text { heck is going wrong here, and then I felt much more empowered to take charge of my health." }\end{array}$ \\
\hline & $\begin{array}{l}\text { "I'm positive definitely because... it gives you validation... but I do feel like it is just one little piece of the puzzle... and I hope that they } \\
\text { don't stop here..., learning about this one mutation because I think there's a lot in the different methylation cycles that will be able to give } \\
\text { us more answers... and help us get better. That's what l'm hoping for. I'm excited about knowing more." }\end{array}$ \\
\hline \multirow[t]{2}{*}{ Confused/Overwhelmed } & "I've had enough, you know, it's overwhelming so that's the part that's difficult, I think, to try and synthesize and interpret all the information." \\
\hline & $\begin{array}{l}\text { "I think for me it's that there's not enough knowledge out there for to help us and really... I mean there have been doctors don't even know } \\
\text { it until you bring it up to them." }\end{array}$ \\
\hline \multirow[t]{2}{*}{ Indifferent } & $\begin{array}{l}\text { "It didn't concern me too much... I was glad to know it because [it's] another piece of information that you can have for future. It wasn't a } \\
\text { deal to me ...It's pretty common so... it's kind of like well, what else is new?" }\end{array}$ \\
\hline & 2OVIDFR. "Some peonle \\
\hline
\end{tabular}

Table 2: Validating patients' personal health experiences through diagnosis.

For the majority of participants, testing and identification of a genetic polymorphism also conferred a sense of validation of their personal health journey which, to date, had not been explainable with pre-existing biomedical models of disease. While validating, the emotional timbre illustrated in the quotes in Table 2 is both positive and negative (Table 2).

\section{Presenting symptoms}

Symptomatology of MTHFR polymorphisms was the second major domain of inquiry. Presenting symptoms are highly heterogenous. Patients and providers generally agreed that presenting symptoms were heterogenous and unpredictable. Presenting symptoms that were notably different between patients and providers included: fatigue, hormones, GI, allergies, insomnia, anxiety, brain fog, and poor memory. Patients and providers reported similar proportions of presenting symptoms such as neurological manifestations, migraines, and depression. Figure 2 summarizes the frequency of presenting symptoms by category. Note the categorization in Figure 2 represents patient and provider reports and thus some are very specific, while others very general.

\section{Characteristics of treatment protocol}

Both patients and doctors struggled to describe specific treatment 


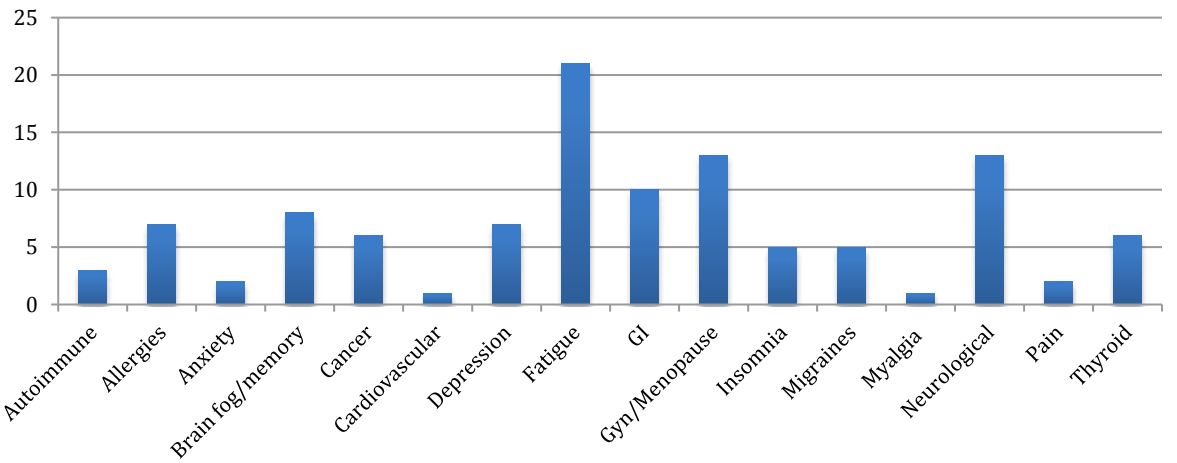

Figure 2: Frequency of Presenting MTHFR Symptoms

protocols. Treatment regimens were highly individualized. As one doctor summarized: "Everybody's treatment protocol is so different, I think that's why it's hard to basically outline or say this is the protocol we do for each person because everybody's physiology is so different in how they respond."

Patients also reported modifying the frequency of treatment, including injections, on their own based on their symptom experiences. However, a few pearls were gained regarding preferred protocols. The vast majority of patients and providers reported optimal results using a combination of methylfolate plus methylcobalamin in the injectable form. Methylcobalamin alone was used as initial treatment by a third of participants; however methylfolate was added when insufficient response was achieved with methylcobalamin alone.

Patient 1: "So I was pretty much on that same diet and the methyl B12 before I started the folate, so it makes a difference. The folate made the difference."

Patient 2: "I've taken B12 shots and B12 sublingually for most of my life, and it wasn't until they discovered this methylfolate aspect of it that it really started making a difference... I'm very energized when I have my shots whereas before, I would kind of perk up, [followed by] another crash, so it wasn't really effective"

Reported dosages were highly variable among doctors.

- "I always use a 5:1 cocktail [B12:methyfolate], even when I'm doing pediatrics...I always try to keep the B12 dominant"

- "Injectable works really well in a one-to-one ratio"

- "I think twice a week is a really good maintenance dose of injectable methylfolate-B12."

- [when using a folate and a B12] I start most people off with a oneto-one ratio, 1000 micrograms each"

All of the participants reported differences between oral supplementation and injectable forms of methylfolate. For example, one doctor stated:

"If I can get them to inject, I definitely do, especially if I suspect their gut is a wreck because they're not going to absorb anyway." Another: "I only ever prescribe shots" and "I very rarely do anything orally unless they are having a hard time getting the shots or they live far away, but yeah, the injections are definitely far superior than anything orally"

Similarly, patient comments reflected profound differences between routes of administration: "it did make a huge difference once I started the injections. Like I said, the pills didn't really do anything for me, but once I added the B12 and started going to the injection form, it brought me back. Then I was able to [function] because you get the brain fog so bad that you can't even think to help yourself"

Protocol variations included starting patients on injectable forms for a limited duration, then switching them to oral forms, plus alternative "methyl donors" such as royal bee jelly, choline, and trimethylglycine (TMG) along with methylfolate. Another doctor started patients with a "methyl challenge" described as giving the patient a large dose of both methylcobalamin and methylfolate by injection and monitoring the clinical response. Another doctor's typical approach used the injectable forms concurrently with additional sublingual B-12. A fifth doctor reported choosing l-methylfolate (Deplin ${ }^{\infty}$ brand) on occasion - an approach to which others objected due to the high dose of methylfolate in Deplin, 7 or $15 \mathrm{mg} /$ capsule.

\section{Improvements in Presenting and Other Symptoms}

Twenty-eight (93\%) of participants reported changes in symptoms with methylfolate treatment, including improvements in physical, mental, and other facets of health and well-being. Doctors and patients reported symptom changes differently (Table 3). For example, the most frequent improvement from the patient perspective was an improvement in energy, which was not described by doctors. One participant stated:

"My energy picked up...I'm going to clean my whole house. I've cleaned all my cupboards. I cleaned up all my files, so I'm running out of things to do. You know, I've got so much energy."

Changes in symptoms reported to different degrees by patients and doctors also included cognition and improved sleep. Improved immune function was discussed only by patient participants.

Reported changes in depression and anxiety were also notable. The words of this participant offer insight into the magnitude of her experience:

"I told her [the doctor]: I am so tired. I can't think. I can't sleep. I have insomnia. I don't know where I'm going. You know, the San Francisco bridge is looking pretty good right now."

Another participant, reflecting on changes in mental clarity stated the following:

"I took my first injection, and I was shocked that I had this instant mental clarity" and "I think a lot of it has to do with the injections, and 
Citation: Oberg E, Givant C, Fisk B, Parikh C, Bradley R (2015) Epigenetics in Clinical Practice: Characterizing Patient and Provider Experiences with MTHFR Polymorphisms and Methylfolate. J Clin Med Genom 3: 124. doi: 10.4172/2472-128X.1000124

Page 6 of 8

\begin{tabular}{|c|c|c|c|c|}
\hline Symptom changes & $\begin{array}{l}\text { Code Frequency } \\
(\mathrm{N}=192)\end{array}$ & $\%$ Patient & $\%$ Provider & Symptom Change Quotes \\
\hline Energy & 59 & $31 \%$ & $0 \%$ & $\begin{array}{l}\text { "Once I started on the B vitamins and that folate, I felt amazing." • "My energy picked up. I'm going to } \\
\text { clean my whole house. I've cleaned all my cupboards. I cleaned up all my files, so I'm running out of } \\
\text { things to do. You know, I've got so much energy." • "I have stamina and energy " }\end{array}$ \\
\hline Physical symptoms & 58 & $27 \%$ & $32 \%$ & $\begin{array}{l}\text { "I felt lousy and now I know what it feels like to feel good" • "...time for the shot, and that will help me } \\
\text { get it through Monday and I'll feel great for... probably the whole week, until Friday or so..."• "I'm getting } \\
\text { treated and I'm now feeling } 100 \% \text { better." }\end{array}$ \\
\hline Mood symptoms & 52 & $25 \%$ & $21 \%$ & $\begin{array}{l}\text { "I can say that I'm less moody. I'm not depressed when I'm taking my shots like I should. I'm not on a } \\
\text { rollercoaster." • I'm happier so it hits me on an emotional frequency. I'm clear." }\end{array}$ \\
\hline $\begin{array}{l}\text { Behavioral } \\
\text { symptoms }\end{array}$ & 13 & $6 \%$ & $11 \%$ & $\begin{array}{l}\text { "At one point, I had given up doing the shots because I didn't think it was doing anything, and then [I felt] } \\
\text { a lot worse so I reinstituted... the depression is better, but I still have a lot of issues with focus, and... } \\
\text { ADD... I'm in office doing a little thing here...and the chair is just turning around to all sides of my desk, } \\
\text { and then } 8 \text { hours have passed..." • "my husband [has] moods like I've never seen...really swings [and } \\
\text { has] bouts of anger, and it'd gotten better, but he's not on the full regime yet" }\end{array}$ \\
\hline No change & 13 & $6 \%$ & $5 \%$ & $\begin{array}{l}\text { "I've been taking [methylfolate and B12], but to be honest with you, I'm really not sure if it's doing anything } \\
\text { for me. I haven't seen any real significant changes or improvements, but it's hard to say because, you } \\
\text { know, I've got so many other chemicals in me too in addition to the B12 that I really don't know for sure" } \\
\text { - "I really haven't noticed a difference, but then again, I'm on other types of medication now ... and so I } \\
\text { can't see an overall result of just from the B12 shots." }\end{array}$ \\
\hline Mental symptoms & 12 & $6 \%$ & $5 \%$ & $\begin{array}{l}\text { "It did make a huge difference at once... I added the B12 and started getting the injection form. It brought } \\
\text { a lot back, and then I was able to [function] because you get the mind fog so bad that you can't even } \\
\text { think to help yourself" • "I didn't notice that [testosterone] helped my energy anywhere near as much as } \\
\text { the B12 and folate. I noticed that my thinking is clear I'll go to work and I'm able to concentrate. I'm able } \\
\text { to get things done quickly..." }\end{array}$ \\
\hline Improved sleep & 5 & $3 \%$ & $0 \%$ & "'I haven't slept for years and I'm sleeping much better now for these last 3 weeks." \\
\hline Improved immunity & 3 & $<1 \%$ & $0 \%$ & $\begin{array}{l}\text { "I got severe flu-like symptoms any time I exercised and I've always been an avid exercise person, and it } \\
\text { got to a point where I was in bed, sick, fever, everything... this is all what has been prevent[ed] from the } \\
\text { injections" •" I've really noticed just a lot in not getting the little colds and then the } 2 \text {-month long flu and } \\
\text { just all that kind of stuff." • "I just feel a lot better, and I rarely get sick anymore...I feel like my immune } \\
\text { system is much stronger." }\end{array}$ \\
\hline
\end{tabular}

Table 3: Symptoms changes following treatment with methylfolate-methylcobalamin

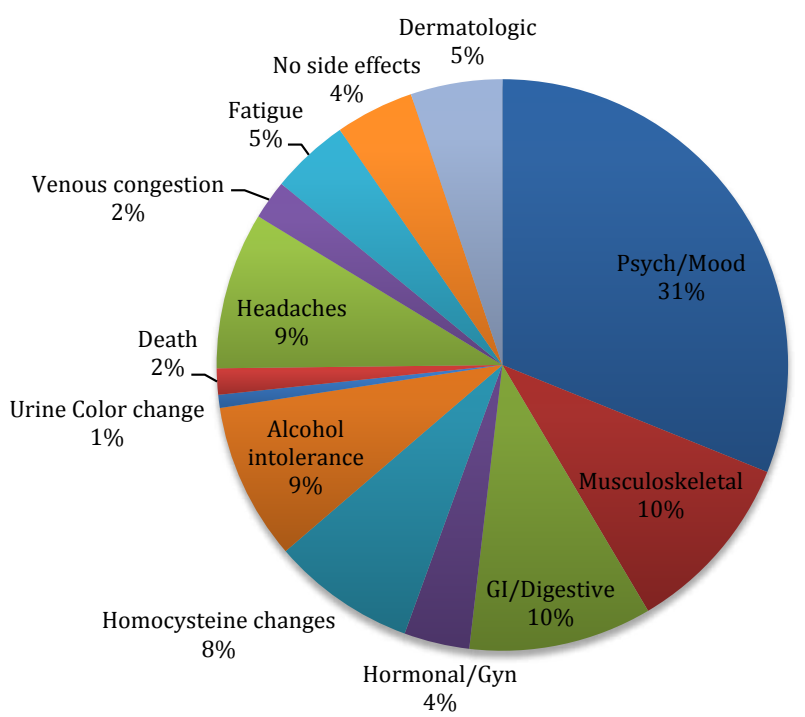

- Psych/Moo

- Musculoskeletal

GI/Digestive

- Hormonal/Gyn

- Homocysteine changes

- Alcohol intolerance

urine Color change

Death

Headaches

Venous congestion

Fatigue

No side effects

Dermatologic

Figure 3: Side Effects of Methylfolate-Methylcobalamin Treatment (Drs \& Pts)

I think clearly now, and I'm not flying off the handle. I don't jump, you know, react on impulse. I actually can take it in, take a deep breath, smell the roses like they say, and then go 'okay'" (Table 3).

\section{Adverse Events and Side Effects}

As might be expected, not all symptom experiences were positive and reports of side effects and adverse events were as heterogeneous as the reported symptoms improvements. While most side effects were minor, some were severe. Types and prevalence of side effects are detailed in Figure 3.

One hypothesis emerged through doctor reports; side effects may be due to "over methylation". Regarding over methylation, one doctor reported:

"It's pretty quick when it happens. I would say it's within a week that you'll see over-methylation in a sensitive patient." This doctor attributed gastro-intestinal symptoms and increased anxiety to over-methylation. 
Several patient comments also support this observation: "I think I get too hyped up and I feel like I can't go to sleep at night and I get nervous."

\section{An unexpectedly reported side effect was alcohol intolerance:}

"For me, I think one [side effect] is alcohol [related]. I'm not able to tolerate any alcohol... This weekend, we had a barbecue. I had one small drink. The next day, I had to sleep all day."

\section{Discussion}

The major themes explored in this qualitative study included: 1) characteristics of clinical treatment 2) reactions to diagnosis, 3) reports of presenting physical and mental symptom 4) changes in presenting and other signs and symptoms, and 5) side effects and adverse events. Both patients and participants provided clinically relevant information in each of these themes. Most remarkably, the reported heterogeneity in all themes demonstrates the need for standardized research. For example, more granular research is necessary to report the prevalence of under-recognized signs and symptoms that may indicate MTHFR polymorphism prior to the onset of diagnosable chronic diseases like depression and cardiovascular disease. Similarly, doctors should be educated on the emotionality patients experience upon diagnosis, and be prepared with genetic counseling resources for patients upon request. The diversity of clinical treatment protocols elucidated by our interviews also points to the need for controlled research to determine the optimal composition, dose and duration of treatment protocols, including continued research on the role of methylfolate and the significance of route of administration on clinical response. Finally, perhaps most importantly, detailed evaluations of the risk to benefit ratio of physical, mental and emotional "improvements" compared to adverse effects should be formally characterized in larger samples. Although the majority of participants reported generally beneficial effects (exemplified by their voluntary continuation of treatment), several severe adverse effects were described as possibly attributable to "treatments".

In general, we were most surprised with the lack of consistency in treatment approaches. Clinicians appear to be relying largely on trial and error dosing schedules both in terms of quantity and frequency. Furthermore, we learned that patients were modifying their own dosing regimens and not always informing their clinicians, suggesting there is substantial room for improvement in patient-provider communication. Similarly, the range and diversity of side effects was heterogenous. There appeared to be differences in the side effects of which clinicians were aware and were monitoring for, and the total range of side effects that patients report. Compounded with the variability in treatment protocols, it is difficult to interpret which side effects are common with a "standard" treatment protocol and which may be more common as symptoms of overdosing specific agents, or are unrelated to treatments. These elements pose a challenge for trial design in terms of developing an appropriate protocol, but indicate a strong need for randomized trials with thoughtful controls and detailed adverse event monitoring.

Despite this relatively chaotic portrayal of current clinical practices, patients overwhelmingly reported positive, profound experiences. They experienced life-transforming changes in energy, chronic depression, fatigue, and other complex, vague symptoms that challenge clinicians. A sense of validation was common, although sometimes tinged with regret about the time spent feeling ill prior to identification of their MTHFR polymorphism. Yet, a few participants also reported neutral experiences, suggesting methylfolate and/or methylcobalamin may result in clinically meaningful responses in certain patterns of MTHFR polymorphism.
The experiences of confusion and the expressed concerns about family members' status exemplifies the need for appropriate provider training in genetic counseling and/or the need to have referral resources available in practices conducting genetic testing. Genetic counseling was once primarily reserved for prenatal counseling or in response to serious heritable conditions. Now, it is available direct-to-consumer over the internet. The patients we interviewed reported that they did not necessarily know they were undergoing genetic testing, and did not fully understand or appreciate the implications when results were reported. The ethical guidelines around informed consent regarding genetic testing and the rights and responsibilities of family members has been a topic in literature[27]. Furthermore, provider education and training in doctor-patient communication and strategies to convey sensitive health information would be beneficial.

Our research has limitations. Inter-rater reliability was not assessed prior to coding the focus group transcripts, however, a retrospective review of coding revealed good consistency between coders. As with any qualitative research involving focus groups, some responses may be influenced by the group setting; we attempted to control for this by tracking response saturation. Some topics, such as alcohol intolerance, emerged in only one group, although the experience was shared by several respondents. Similarly, some of the differences between patient and provider reporting of symptoms could be due to differences in the natural course of conversation in particular focus groups. Provider perspectives on patients' symptoms are also limited by what patients report. Additionally, patients are more likely to remember their own medical history, and thus provider recollection of patient symptoms may not be as accurate unless relevant to patients' chief complaints

Strengths of this study include that this is the first systematic report of patient and provider experiences with methylfolate and MTHFR polymorphisms and therefore it adds a scientific, semi-quantitative perspective to the crowd-sourced experiences shared on internet forums. It is the first report of treatment protocols in use by clinicians, as well as, the first formal documentation of side effects potentially from methylfolate treatment. Thus the results will inform future clinical trial design and safety surveillance in further research.

\section{Conclusion}

Evaluation of MTHFR polymorphisms is becoming increasingly common in clinical practice, however is being implemented without careful consideration to patient communication, treatment adherence, detailed documentation of clinical response including side effects, and diversity of treatment protocols without validation. All of these limitations in current practice have the potential to risk patient access to potentially life-altering diagnostic information, as well as, potentially efficacious nutritional treatments. Future controlled research is critical to advancing nutrigenomics in clinical practice. Additionally, safety surveillance employing provider research networks may be a feasible intermediate safety measure to consider in this rapidly advancing, experimental practice.

\section{Conflicts of Interest}

This research was supported by LaVita Compounding Pharmacy.

\section{References}

1. S. Friso S, Udali P, Guarini C, Pellegrini P, Pattini S, et al. (2013) Global DNA hypomethylation in peripheral blood mononuclear cells as a biomarker of cancer risk. Cancer Epidemiol Biomarkers 22: 348-55.

2. Rozen R (2000) Genetic modulation of homocysteinemia. Semin Thromb Hemost 26: 255-261. 
Citation: Oberg E, Givant C, Fisk B, Parikh C, Bradley R (2015) Epigenetics in Clinical Practice: Characterizing Patient and Provider Experiences with MTHFR Polymorphisms and Methylfolate. J Clin Med Genom 3: 124. doi: 10.4172/2472-128X.1000124

3. Födinger M, Hörl WH, Sunder-Plassmann G (2000) Molecular biology of 5,10-methylenetetrahydrofolate reductase. J Nephrol 13: 20-33.

4. Botto LD, Yang Q (2000) 5,10-Methylenetetrahydrofolate reductase gene variants and congenital anomalies: a HuGE review. Am J Epidemiol 151: 862 877.

5. Bailey LB, Gregory JF 3rd (1999) Polymorphisms of methylenetetrahydrofolate reductase and other enzymes: metabolic significance, risks and impact on folate requirement. J Nutr 129: 919-922.

6. Reilly R, McNulty H1, Pentieva K1, Strain JJ1, Ward M1 (2014) MTHFR 677TT genotype and disease risk: is there a modulating role for B-vitamins? Proc Nutr Soc 73: 47-56

7. L Kevere, S Purvina, D Bauze, M Zeibarts, R Andrezina, et al. (2014) Homocysteine and MTHFR C677T polymorphism in children and adolescents with psychotic and mood disorders. Nord J Psychiatry 68: 129-36.

8. Lewis SJ, Ebrahim S, Davey Smith G (2005) Meta-analysis of MTHFR 677C->T polymorphism and coronary heart disease: does totality of evidence support causal role for homocysteine and preventive potential of folate? BMJ 331: 1053

9. CABousman, M Potiriadis, IP Everall, JM Gunn (2014)Methylenetetrahydrofolate reductase (MTHFR) genetic variation and major depressive disorder prognosis: A five-year prospective cohort study of primary care attendees. Am J Med Genet B Neuropsychiatr Genet 68-76.

10. NC Htun, K Miyaki, C Zhao, M Muramatsu, N Sato (2014) Epistasis effects of COMT and MTHFR on inter-individual differences in mental health: under the inverted U-shaped prefrontal dopamine model. Biochem Biophys Res Commun 451: $574-579$.

11. Gilbody S, Lewis S, Lightfoot T (2007) Methylenetetrahydrofolate reductase (MTHFR) genetic polymorphisms and psychiatric disorders: a HuGE review. Am J Epidemiol 165: 1-13.

12. Frustaci A, Neri M, Cesario A, Adams JB, Domenici E, et al. (2012) Oxidative stress-related biomarkers in autism: systematic review and meta-analyses. Free Radic Biol Med 52: 2128-2141.

13. Obeid , Holzgreve W, Pietrzik K (2013) Is 5-methyltetrahydrofolate an alternative to folic acid for the prevention of neural tube defects? J Perinat Med 41: $469-483$

14. MV Holmes, $P$ Newcombe, JA Hubacek, $R$ Sofat, SL Ricketts, et al. (2011) Effect modification by population dietary folate on the association between MTHFR genotype, homocysteine, and stroke risk: a meta-analysis of genetic studies and randomised trials. Lancet 378: 584-94.

15. Q Yang, L Bailey, R Clarke, WD Flanders, T Liu, et al. (2012) Prospective study of methylenetetrahydrofolate reductase (MTHFR) variant C677T and risk of allcause and cardiovascular disease mortality among $6000 \mathrm{US}$ adults. Am J Clin Nutr 95: 1245-53.

16. Kang S, Wu Y, Liu L, Zhao X, Zhang D2 (2014) Association of the A1298C polymorphism in MTHFR gene with ischemic stroke. J Clin Neurosci 21: 198202.
17. Qian X, Lu Z, Tan M, Liu H, Lu D (2007) A meta-analysis of association between C677T polymorphism in the methylenetetrahydrofolate reductase gene and hypertension. Eur J Hum Genet 15: 1239-1245.

18. Friso S, Girelli D, Trabetti E, Olivieri O, Guarini P, et al. (2005) The MTHFR $1298 \mathrm{~A}>\mathrm{C}$ polymorphism and genomic DNA methylation in human lymphocytes. Cancer Epidemiol Biomarkers Prev 14: 938-943.

19. CH Pullin, PA Ashfield-Watt, ML Burr, ZE Clark, MJ Lewis, et al. (2001) Optimization of dietary folate or low-dose folic acid supplements lower homocysteine but do not enhance endothelial function in healthy adults, irrespective of the methylenetetrahydrofolate reductase (C677T) genotype. J Am Coll Cardiol 38: 1799-805.

20. PA Ashfield-Watt, CH Pullin, JM Whiting, ZE Clark, SJ Moat, et al. (2002) Methylenetetrahydrofolate reductase $677 \mathrm{C}-->\mathrm{T}$ genotype modulates homocysteine responses to a folate-rich diet or a low-dose folic acid supplement: a randomized controlled trial. Am J Clin Nutr 76: 180-186.

21. KS Crider, JH Zhu, L Hao, QH Yang, TP Yang, et al. (2011) MTHFR 677C->T genotype is associated with folate and homocysteine concentrations in a large, population-based, double-blind trial of folic acid supplementation. Am J Clin Nutr 93:1365-72.

22. Haggarty P1, McCallum H, McBain H, Andrews K, Duthie S, et al. (2006) Effect of $B$ vitamins and genetics on success of in-vitro fertilisation: prospective cohort study. Lancet 367: 1513-1519.

23. Alpert JE1, Mischoulon D, Rubenstein GE, Bottonari K, Nierenberg AA, et al (2002) Folinic acid (Leucovorin) as an adjunctive treatment for SSRI-refractory depression. Ann Clin Psychiatry 14: 33-38.

24. http://mthfr.net

25. SVIGK Rosenstock IM, Lewis FM, Rimer BK (1997) The health belief model. San Francisco: Jossey-Bass.

26. Elo S, Kyngäs H (2008) The qualitative content analysis process. J Adv Nurs 62: $107-115$

27. A Harris, SE Kelly, S Wyatt (2013) Counseling customers: emerging roles for genetic counselors in the direct-to-consumer genetic testing market. J Genet Couns 22: 277-88 